\title{
深层油气藏多级迂回暂堵压裂技术研究
}

\author{
蒋廷学 $^{1,2}$, 下晓冰 ${ }^{1,2}$ \\ 1) 页岩油气有效开发国家重点实验室，北京 102206 ; \\ 2) 中国石油化工股份有限公司石油工程技术研究院，北京 102206
}

摘 要: 中国埋深超过 $3500 \mathrm{~m}$ 的深层油气资源量丰富, 但深层油气藏水平井分段压裂面临裂缝净压 力小、压力窗口有限的难题, 导致常规暂堵压裂难以实施. 本研究提出多级迁回暂堵压裂的技术思路, 建 立相应的数学模型并采用牛顿迭代法进行求解, 并对迁回暂堵的时机和降排量幅度等工艺参数进行模拟及 优化. 结果表明, 迂回暂堵可以较大幅度地提高压裂施工压力窗口及主裂缝的净压力, 有助于实现深层-超 深层油气藏压裂裂缝的转向及改造体积的大幅度提高; 多级迂回暂堵压裂可在单级迂回暂堵压裂的基础上, 进一步增加压裂施工压力窗口及主裂缝净压力, 有助于实现深层-超深层油气藏压裂裂缝复杂性程度及改造 体积的最大化; 其他工艺成本包括压裂车组等的费用基本维持不变. 示例井应用效果证明, 压后产量可比 邻并提高 $30 \%$ 以上. 因此, 多级迁回暂堵压裂技术对提高深层及超深层油气藏的压裂效果、开发水平及经 济效益等，都具有重要的指导意义和应用价值.

关键词：深层油气藏；低渗透储层；水平井；迂回暂堵；多级压裂；压裂参数

中图分类号：TE348；TE358 文献标志码：A doi: 10.3724/SP. J. 1249.2021.06590

\section{Multistage circuitous-temporary plugging fracturing technology for deep oil-gas reservoirs}

\section{JIANG Tingxue ${ }^{1,2}$ and BIAN Xiaobing ${ }^{1,2}$}

1) State Key Laboratory of Shale Oil and Gas Enrichment and Mechanisms and Effective Development, Beijing 102206, P. R. China

2) Sinopec Research Institute of Petroleum Engineering, Beijing 102206, P. R. China

\begin{abstract}
China is rich in deep oil and gas resources with buried depth of more than $3500 \mathrm{~m}$. To overcome the technical difficulties in the implementation of conventional temporary plugging technology for the staged fracturing of horizontal well of deep oil-gas reservoirs, such as the small net pressure of fractures and the limited pressure window, we propse a technical idea of multi-level circuitous temporary plugging of fracturing and then build a mathematical model and solve this model by Newton iterative method. On this basis, we conduct the substantive research work on the simulation and optimization of technical parameters, such as the timing of circuitous temporary plugging, the decrease of pump rates, etc. The research results show that circuitous temporary plugging can significantly improve the pressure window of fracturing construction and the net pressure of the main fracture, which is helpful to realize the diversion of the fractures of deep and ultra-deep oil-gas reservoir and greatly increase the reformed volume; multistage circuitous temporary plugging fracturing can further increase the fracturing pressure window and the net
\end{abstract}

Received: 2021-07-29; Accepted: 2021-09-04; Online (CNKI) : 2021-10-13

Foundation: National Natural Science Foundation of China (U19B6003) ; Sinopec Science and Technology Project (P20046-3)

Corresponding author: Professor JIANG Tingxue. E-mail: jiangtx.sripe@ sinopec.com

Citation: JIANG Tingxue, BIAN Xiaobing. Multistage circuitous-temporary plugging fracturing technology for deep oil-gas reservoirs $[\mathrm{J}]$. Journal of Shenzhen University Science and Engineering, 2021, 38(6) : 590-597. (in Chinese)

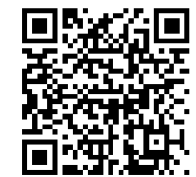


pressure of the main fracture based on single-level circuitous temporary plugging, which is conducive to achieving the complexity of the fractures of deep and ultra-deep oil-gas reservoir and the maximization of the reformed volume. And meanwhile remaining the cost of fracturing vehicles and other processes basically unchanged. The application results of test wells show that the production after fracturing can be increased by more than $30 \%$ compared with adjacent wells. Therefore, the multi-level circuitous temporary plugging technology provides important guiding significance and reference for improving the fracturing effect, exploitation level and economic benefits of deep-ultra-deep oil-gas reservoir.

Key words: deep buried reservoir; low permeability layer; horizontal well; circuitous temporary plugging; multi-stage hydraulic fracturing; fracturing parameter

随着页岩气勘探开发的突破，水平井体积压裂 技术已逐步向 “密切割、强加砂、暂堵转向” 等方 向转变. 其中, 暂堵转向是核心, 它包含两个层 次: 一是井筒的暂堵, 关系到多簇射孔是否能全部 产生裂缝; 二是裂缝内暂堵, 关系到转向支裂缝能 否形成, 以及裂缝的复杂性程度能有多大幅度的提 升等. 本研究主要探讨裂缝内的暂堵转向问题, 这 是体积压裂能否成功的技术关键.

北美 Wolfcamp 页岩采用集群优化实施多级暂 堵转向压裂优化, 形成了暂堵剂实验评价、裂缝扩 展数值模拟和现场作业及数据分析流程, 通过优化 策略优选出了井工厂多口井之间采用非暂堵和暂堵 交替压裂工艺 ${ }^{[1-2]}$. 通过压力监测, 对水平井暂堵 时的压力响应进行统计, 以压力响应达到 $3.4 \mathrm{MPa}$ 作为暂堵转向效果良好的临界值, 取得了较好的应 用效果 ${ }^{[2]}$. 中国以长庆油田、吉木萨尔油田、长宁 页岩气田和涪陵页岩气田等为代表, 已经开展了多 级暂堵压裂技术应用, 初步建立了耦合井筒流场及 暂堵球、暂堵剂运动的数值模拟方法, 优化了现有 双暂堵压裂工艺的部分参数, 长宁、涪陵单段暂堵 级数最高达 $2 \sim 4$ 级, 实现裂缝的多次转向, 进而 构建复杂缝 ${ }^{[3-4]}$.

多级暂堵技术在理论上可实现储层的均匀充分 改造, 但现场作业流程仍以经验判断居多, 现有工 艺仍存在技术局限性. 尤其随着储层埋深的不断增 加, 井口施工压力越来越高, 有时正常的施工压力 距离施工限压设计值已相对很小 (小于 $5 \mathrm{MPa}$ ), 此时在有限的压力窗口下, 已不能进行正常的缝内 暂堵设计及作业. 深层压裂的两向水平应力差一般 相对较大, 裂缝的复杂性程度随埋深的增加逐渐降 低, 因此, 更迫切需要缝内暂堵以实现深层复杂裂 缝甚至体积裂㖓的技术需要. 为此, 本研究提出了 迂回暂堵或多次迁回暂堵工艺技术.

迂回暂堵是指当压力窗口太小时, 适当降低排
量, 此时因排量降低引起的井筒沿程摩阻和裂缝摩 阻会相应降低, 从而提高压力窗口. 排量的降低并 不影响暂堵裂缝的憋压或缝内净压力的提升, 裂缝 在宽度方向上的进一步增加引起进缝摩阻降低, 由 此可再逐步提升排量, 甚至可恢复原先的排量水 平. 即, 迂回暂堵在压力窗口受限的前提下仍可实 现通过暂堵提高缝内净压力的目标, 进而实现深层 复杂裂缝的技术目标. 而多级迂回暂堵压裂可对单 级迂回暂堵的净压力增加具有逐次叠加效应, 以实 现单级暂堵压裂实现不了的技术目标. 如能在裂缝 不同位置处都进行上述迁回暂堵施工, 则可以在深 层主裂缝范围内提高转向支裂缝的分布密度, 并实 现大幅度提升裂缝复杂性及改造体积的技术目标.

本研究通过对迂回暂堵的时机、降排量幅度、 对应的缝宽及净压力变化等进行系统模拟, 优化了 迂回暂堵工艺参数, 并结合部分实际案例进行了分 析讨论, 对深层油气藏实现体积压裂有重要的指导 意义.

\section{1 多级迁回暂堵压裂的数学模型}

基于多级迁回暂堵压裂理论, 建立了相应的数 学模型. 模型的假设条件为: (1) 岩石是均质, 且 各向同性的线弹性体, 水力裂缝垂直横截面满足弹 性力学平面应变条件; (2) 压裂液为幂律性流体, 不考虑压裂液压缩性; (3) 不考虑温度和化学作用 对压裂液性质的影响.

\section{1 模型的基本方程、初始条件及边界条件}

1) 某簇裂缝内流体流动方程

$$
\frac{\partial p_{i}}{\partial L}=-\frac{12}{\Phi} \frac{q_{i} \mu}{w^{3} H}
$$

其中, $p_{i}$ 为某簇裂缝的缝内压力; $q_{i}$ 为某簇裂缝的缝 内流量; $\mu$ 为压裂液黏度; $w$ 为裂缝宽度; $H$ 为裂缝 高度; $\Phi$ 为形状因子; $L$ 为裂缝长度. 
2）连续性方程 ${ }^{[5-7]}$

在裂缝内存在:

$$
\left\{\begin{array}{l}
-\frac{\mathrm{d} q_{i}}{\mathrm{~d} L}=\int_{0}^{L} \frac{2 H C_{\mathrm{L}}}{\sqrt{t-\tau(x)}} \mathrm{d} x+\frac{\mathrm{d} w}{\mathrm{~d} t} \\
t_{0} \leqslant t \leqslant t_{\max }
\end{array}\right.
$$

其中, $C_{\mathrm{L}}$ 为滤失系数; $t$ 为压裂施工时间; $t_{0}$ 为开始 时刻; $t_{\text {max }}$ 为终止时间; $x$ 为裂缝上某一点处的长度; $\tau(x)$ 为 $x$ 处压裂液开始漏失的时间.

井筒内人口流量为

$$
Q_{\text {in }}=\sum_{i=1}^{n} Q_{i}
$$

其中, $Q_{i}$ 为第 $i$ 条裂缝的进液流量.

3）裂缝宽度方程

$$
w=-16 \frac{1-v(z)}{E(z)} \int_{|z|}^{H} \frac{F(\tau)+z G(\tau)}{\sqrt{\tau^{2}-z^{2}}} \mathrm{~d} \tau
$$

其中, $E(z)$ 和 $v(z)$ 为不同深度的弹性模量及泊松 比; $F(\tau)$ 和 $G(\tau)$ 为关于时间的函数 ${ }^{[8]}$.

4) 井筒内压力分布

沿程摩阻 $\Delta p^{[9]}$ 为

$\Delta p=2 f \frac{\rho \bar{v}^{2} L}{\bar{d}}\left(1-R_{\mathrm{fr}}\right)$

其中, $R_{\mathrm{fr}}$ 为降阻率; $\bar{v}$ 为井筒内液体的平均流速; $\bar{d}$ 为井筒内平均直径; $f$ 为摩阻系数.

井口压力 $p_{\mathrm{w}}$ 为

$p_{\mathrm{w}}=p_{\mathrm{n}}+\sigma_{\min }+\Delta p+p_{\mathrm{p}}-p_{\mathrm{h}}$

其中, $p_{\mathrm{n}}$ 为缝内净压力 $; p_{\mathrm{h}}$ 为液柱压力 $; \sigma_{\text {min }}$ 为最小 水平主应力; $p_{\mathrm{p}}$ 为孔眼摩阻.

5) 孔眼摩阻 $p_{\mathrm{p}}$ 为

$$
p_{\mathrm{p}}=\frac{8 \rho Q_{i}^{2}}{\pi^{2} n_{\mathrm{p}}^{2} C d_{\mathrm{p}}^{4}}
$$

其中, $C$ 为孔眼流量系数; $d_{\mathrm{p}}$ 为射孔孔眼直径; $n_{\mathrm{p}}$ 为 射孔孔数.

6) 暂堵前净压力为

$p_{\mathrm{n}}=\left[\frac{E^{4} \mu Q_{\text {in }} V}{\left(1-v^{2}\right)^{4} H^{6}}\right]^{0.2}$

暂堵后净压力 ${ }^{[10]}$ 为

$p_{\mathrm{n}}^{\prime}=\frac{E}{H}\left(\mu L \sqrt{Q_{\text {in }}}\right)^{\frac{1}{3}}$

其中, $V$ 为压裂液体积; $E$ 为杨氏模量; $v$ 为泊松比.

7) 模型的初始条件为

$$
\left\{\begin{array}{l}
\left.w\right|_{t=0}=0 \\
\left.L\right|_{t=0}=0 \\
\left.p\right|_{t=0}=\sigma_{\min }
\end{array}\right.
$$

其中, $p$ 为缝内压力.

8) 模型的边界条件

暂堵前, 人口边界条件为: $Q_{\mathrm{w}}=Q_{\text {in }}$, 缝端边界 条件为: $p_{\text {tip }}=\sigma_{\text {min }}$; 暂堵后, 入口边界条件为: $Q_{\mathrm{w}}=$ $Q_{\text {in }}$, 缝端边界条件为 : $q_{\text {tip }}=0$. 其中, $Q_{\mathrm{w}}$ 为井口注人 排量, $p_{\text {tip }}$ 为缝端压力; $q_{\text {tip }}$ 为缝端流量.

\section{2 求解方法}

考虑井筒摩阻及孔眼摩阻等复杂因素的多缝同 步扩展数学方程组为非线性方程组, 需通过迭代数 值解法才能获得求解. 考虑到该方程组求解的关键 是各射孔簇进液量的流量分配, 可先假设一个多缝 流量的初始分布, 并计算该条件下的缝内压力分 布, 然后根据全井筒连续性方程及井口压力一致的 原则, 基于牛顿迭代法反复迭代, 调整多族裂缝的 流量分布, 直到获得满足精度要求的解, 再进人下 一时间步的计算.

\section{2 常规暂堵压裂工艺参数优化}

常规暂堵压裂主要指暂堵后一直保持恒定的排 量, 施工压力窗口窄, 可能无法实现净压力持续增 长并超过原始水平应力差的目标. 常规暂堵压裂参 数优化主要包括暂堵位置、暂堵剂密度、粒径及组 合与造缝宽度的匹配关系等.

应用 Meyer 软件模拟不同暂堵剂在裂缝中的浓 度分布及计算相应处的支撑缝宽，如与对应的造缝 宽度相当，则认为暂堵剂在该处实现了暂堵. 将暂 堵剂视作支撑剂, 其他支撑剂全部视作压裂液（支 撑剂浓度参数为 0 ), 则可观察暂堵剂在裂缝中运 移轨迹及最终的浓度分布剖面.

示例井暂堵剂在暂堵处的浓度剖面模拟结果如 图 1 所示. 由图 1 可见, 在裂缝前缘端部、裂缝顶 部及裂缝底部的暂堵剂浓度分布最高, 可实现真正 的全方位封堵, 即裂缝的长度及高度基本停止延 伸，暂堵后再注人的压裂液，只能在裂缝宽度方向

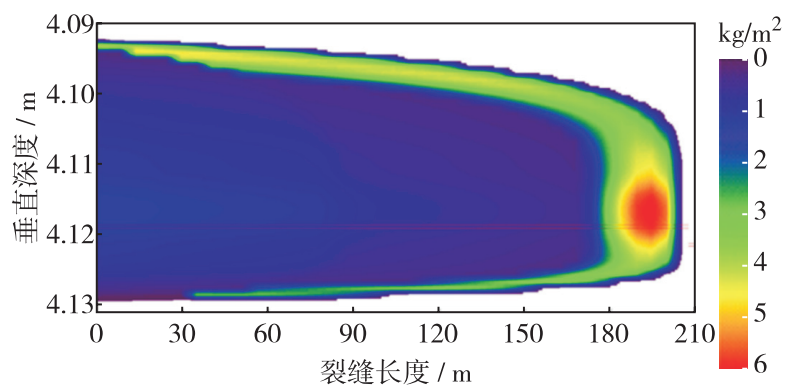

图 1 示例井的暂堵剂浓度剖面

Fig. 1 The profile of temporary agent of the case well 
延伸. 而宽度与缝内净压力呈正相关关系.

结合暂堵剂物理模拟实验, 对暂堵剂的粒径、 浓度、携带液的黏度与排量等与造缝宽度间的匹配 关系进行优化, 结果如图 2. 并将优化结果 ${ }^{[11-12]}$ 与 数模结果进行对比验证.

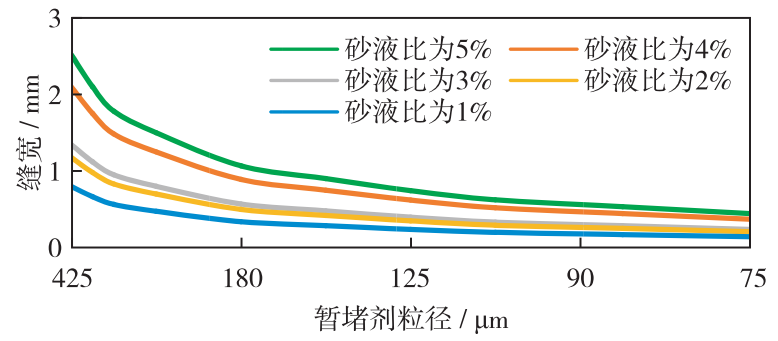

图 2 暂堵剂粒径与裂缝宽度间关系

Fig. 2 The relationships between particle size of temporary agent and crack width

由模拟结果可见，对一定的裂缝宽度而言，要 实现有效封堵, 可采用较小的粒径和较高的砂液比 （支撑剂体积:压裂液体积）组合，也可采用较大的 粒径和较低的砂液比组合. 一般而言, 为提高封堵 效率及降低施工风险，一般采用前者进行封堵.

暂堵后的压力升幅主要以压裂目标井层的原始 水平应力差与暂堵时的净压力差值为最低临界值, 显然低于此临界值的暂堵压裂是无效的，至多促进 了已压开簇裂缝内压裂液及支撑剂的再分配. 但即 便如此, 对促进已压开裂缝簇的均匀进液及均衡进 支撑剂也有一定的正向作用.

\section{3 多级迁回暂堵压裂工艺参数优化}

迁回暂堵包括单级迁回暂堵和多级迁回暂堵两 种方式. 单级迂回暂堵是多级迂回暂堵的基础. 模 拟来自于四川盆地某深层页岩气井.

\section{1 单级迂回暂堵压裂工艺参数优化}

优化的参数主要包括降排量的幅度与对应的裂 缝宽度的动态变化、恢复排量的时机与对应的裂缝 宽度的动态变化、最终能恢复的排量水平，以及缝 内净压力在降排量与恢复排量过程中的动态变化规 律等. 具体模拟结果见图 3 至图 6.

由图 3 模拟结果可见，在单级暂堵条件下，不 同排量降幅后, 缝内净压力及缝宽仍是继续增长 的，且二者增长的趋势基本一致.

由图 4 模拟结果可见，在不同排量的降排量施 工过程中, 地面施工压力仍继续增长, 缝内净压力

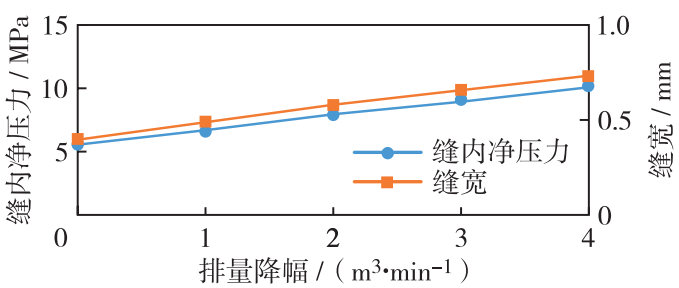

图 3 单级暂堵不同降排量幅度下的 最大动态缝宽及缝内净压力变化

Fig. 3 The change of maximum dynamic width and net pressure with different pump rate decrease under temporary plugging condition

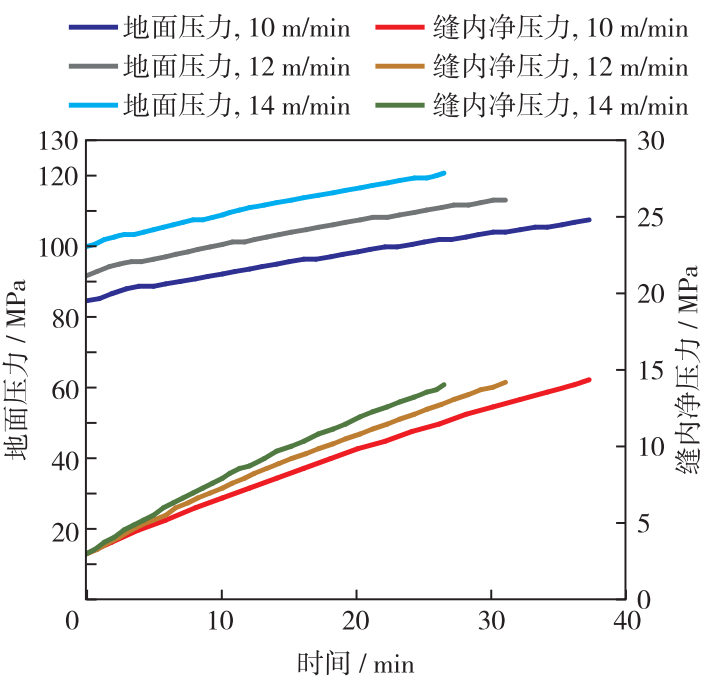

图 4 降排量过程中地面压力和缝内净压力随时间的变化

Fig. 4 Surface pressure and net pressure with time under different pump rates decrease condition

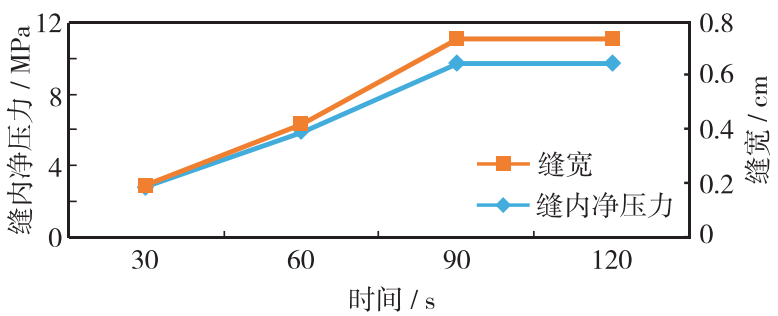

图 5 单级暂堵不同升排量时最大动态缝宽及 缝内净压力变化

Fig. 5 The change of maximum dynamic width and net pressure with different time of increasing pump rate under temporary plugging condition

也是一直持续增长的过程. 由图 5 模拟结果可见， 降排量约 $60 \mathrm{~s}$ 后, 再将排量提升, 净压力的增长速 率更快. 由图 6 模拟结果可见，在同样的压力窗口 下，单级迁回暂堵随时间的延长，净压力增幅 加快. 


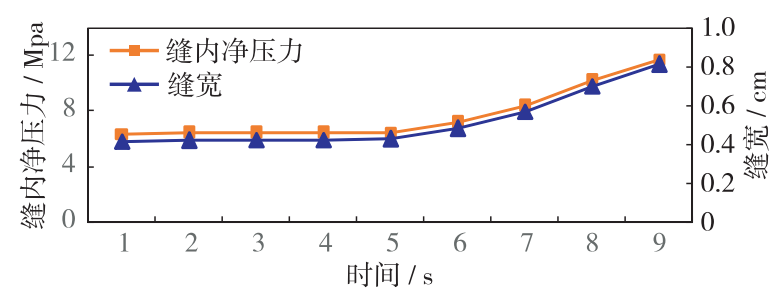

图 6 单级暂堵同样压力窗口下对应的 缝内净压力随时间的变化

Fig. 6 The change of net pressure and time with the same surface pressure limit under temporary plugging condition

\section{2 多级迂回暂堵压裂工艺参数优化}

在单级迂回暂堵的基础上, 如缝内净压力升幅 仍难以突破原始水平应力差值的最低临界值要求, 必须进行 2 级甚至 3 级或以上的多级迁回暂堵压 裂. 基本原理相同, 即通过再次或多次迂回降排量 及后续的升排量操作, 使压力窗口在不增加的前提 下, 不断增加缝内净压力, 直到实现缝内多次裂缝 转向的目标. 但由于起步缝宽不同, 多级迁回暂堵 对应的不同降排量与升排量下的动态缝宽的变化规 律也是不相同的. 与单级暂堵对应的模拟图版分别 见图 7 至图 10.

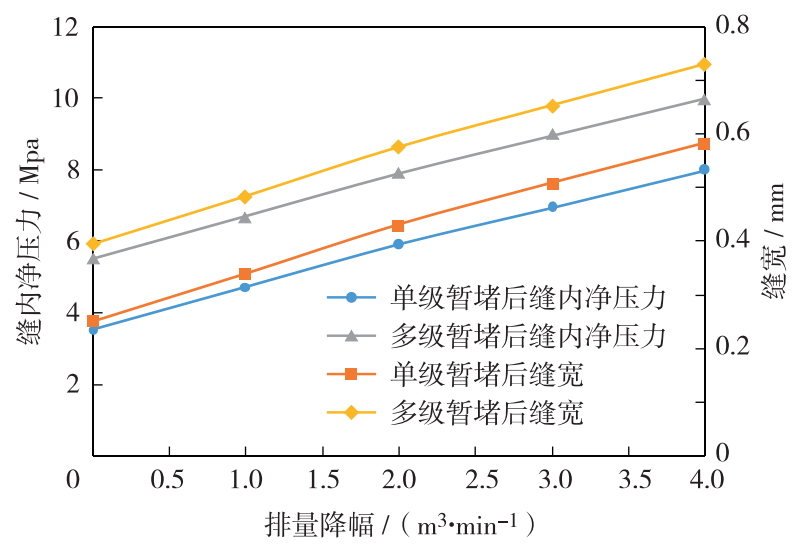

图 7 多级暂堵不同降排量幅度下的 动态缝宽及缝内净压力变化

Fig. 7 The change of dynamic width and net pressure with different pump rates decrease under circuitous temporary plugging condition

由图 7 模拟对比结果可见，在相同的排量降幅 下, 多级暂堵的净压力比单级暂堵的净压力可增加 $50 \%$ 左右.

由图 8 模拟结果可见，在不同排量降幅下，多 级暂堵的地面施工压力与单级暂堵基本重合, 差异
一地面压力, $10 \mathrm{~m} / \mathrm{min}$, 多级暂堵 一 缝内净压力, $10 \mathrm{~m} / \mathrm{min}$, 多级暂堵

一地面压力, $14 \mathrm{~m} / \mathrm{min}$, 多级暂堵 一缝内净压力, $14 \mathrm{~m} / \mathrm{min}$, 多级暂堵

一地面压力, $10 \mathrm{~m} / \mathrm{min}$, 单级暂堵 一缝内净压力, $10 \mathrm{~m} / \mathrm{min}$, 单级暂堵

一地面压力, $14 \mathrm{~m} / \mathrm{min}$, 单级暂堵 一缝内净压力, $14 \mathrm{~m} / \mathrm{min}$, 单级暂堵

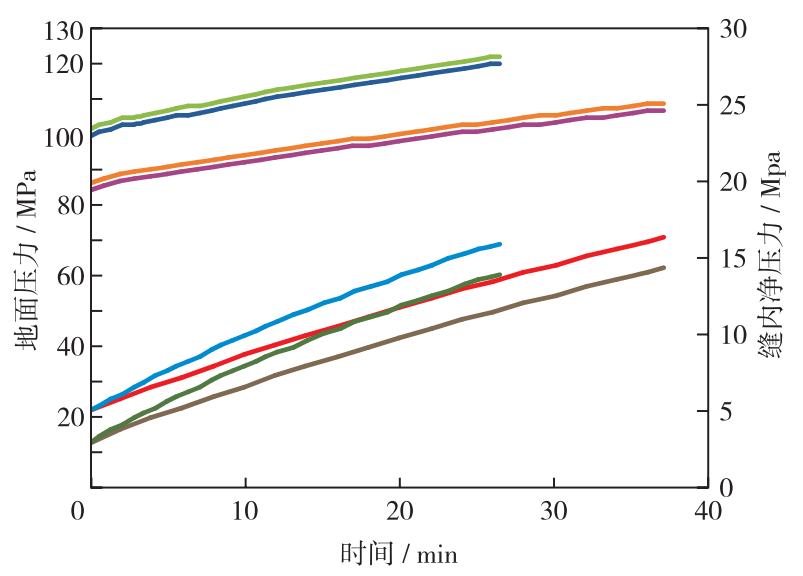

图 8 不同排量下地面压力及缝内净压力随时间的变化

Fig. 8 The change of surface pressure and net pressure with time under different pump rates condition

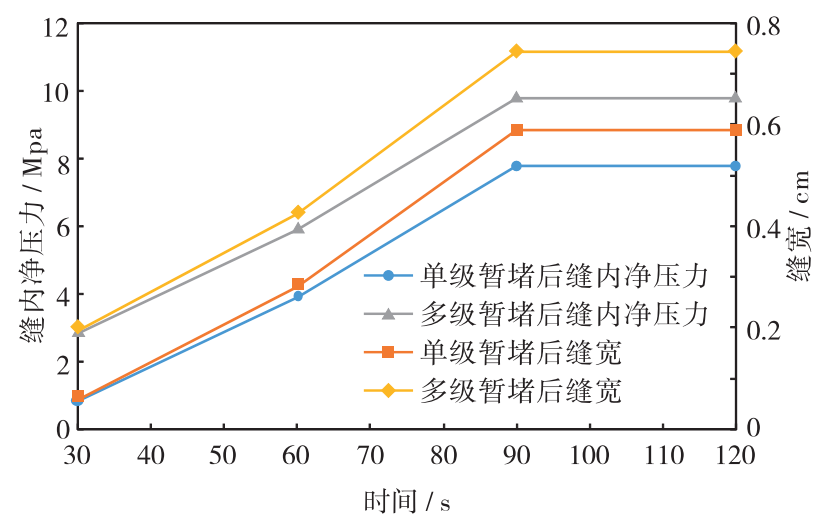

图 9 多级暂堵不同升排量时间动态缝宽及 缝内净压力变化

Fig. 9 The change of dynamic width and net pressure with different time of increasing pump rate under circuitous temporary plugging condition

不大. 说明多级暂堵并未造成压力窗口的损失.

由图 9 可见, 多级暂堵后缝内净压力比单级暂 堵可提高 $30 \%$ 以上. 由图 10 可见，同样压力窗口 下, 多级暂堵后缝内净压力较单级暂堵高 $20 \%$ 左右.

综上所述, 多级迂回暂堵会在上级迂回暂堵的 基础上, 不断增加缝内净压力, 只不过随着暂堵级 数的增加, 缝内净压力增加的幅度会逐渐变缓.

针对暂堵位置, 目前存在近井筒暂堵、中井位 置和缝端位置 3 种情况. 图 11 为不同暂堵位置的 


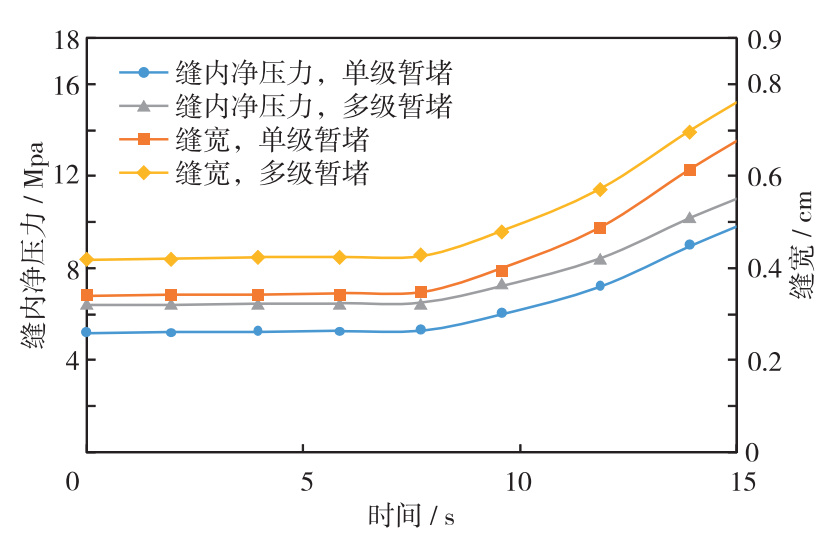

图 10 多级暂堵同样压力窗口下对应的 缝内净压力随时间的变化

Fig. 10 The change of net pressure and time with the same surface pressure limit under circuitous temporary plugging condition

压力响应特征. 在近井筒暂堵特性情况下, 压力上 升速率大, 施工风险高, 但出现支裂㖓少, 支裂缝 的转向半径大. 由于主裂缝扩展早期, 实际上相当 于早期的缝端暂堵. 在中井位置暂堵情况下, 压力 上升速率较大, 施工风险较高, 出现支裂缝也少 (近井支裂缝已饱和), 支裂缝的转向半径大. 由于 主裂缝扩展中期, 实际上相当于中期的缝端暂堵. 在缝端位置暂堵情况下, 压力上升速率适中, 施工 风险较低, 出现支裂缝也少 (近井及中井的支裂缝 都已饱和), 支裂缝的转向半径大 ${ }^{[13-16]}$. 鉴于此, 最佳暂堵位置为缝端. 但也存在裂缝复杂性只局限 于从暂堵位置到井筒的范围内, 裂缝的整体改造体 积相对有限.

\section{4 现场实例分析}

川东南某深层页岩气井 ${ }^{[17-18]}$ 斜深为 $5960 \mathrm{~m}$, 垂深为 $4275 \mathrm{~m}$, 水平段长为 $1504 \mathrm{~m}$, 目的层为五 峰组-龙马溪组, 现场施工压力高、加砂难度大, 部分井通过实施单级及多级迁回暂堵技术, 缝内净 压力增加了 $10 \sim 14 \mathrm{MPa}$, 部分达到 $25 \mathrm{MPa}$ 以上, 顺利完成了全部压裂施工, 测试产量较邻井增加 $30 \%$, 部分增加量甚至达到 1 倍以上.

\section{1 单级迂回暂堵实例分析}

涪陵某口示例井段的单级迁回暂堵压裂施工曲 线见图 11, 该段洜人第 1 个粉砂段塞时将排量提至 $14 \mathrm{~m}^{3} / \mathrm{min}$, 加人 $21 \mathrm{~m}^{3}$ 粉砂段塞后, 施工压力从 $89 \mathrm{MPa}$ 升至 $104 \mathrm{MPa}$ (推测小粒径支撑剂在裂缝中
部及端部形成暂堵), 采取单级迂回降排量至 12.6 $\mathrm{m}^{3} / \mathrm{min}$ 控制后, 压力恢复至 $90 \mathrm{MPa}$ 左右; 在第 2 个粉砂段塞加砂过程中压力又升至 $104 \mathrm{MPa}$, 缝内 净压力增加了 $10 \sim 14 \mathrm{MPa}$ 、增速约为 $1.4 \mathrm{MPa} / \mathrm{min}$ (推测小粒径支撑剂在裂缝端部形成暂堵), 持续降 排量至 $8.5 \mathrm{~m}^{3} / \mathrm{min}$ 后压力平稳下降, 稳定 $5 \mathrm{~min}$ 后 将排量提升至 $12 \mathrm{~m}^{3} / \mathrm{min}$, 后续加砂顺利, 排量恢 复程度达 $85.7 \%$ 。

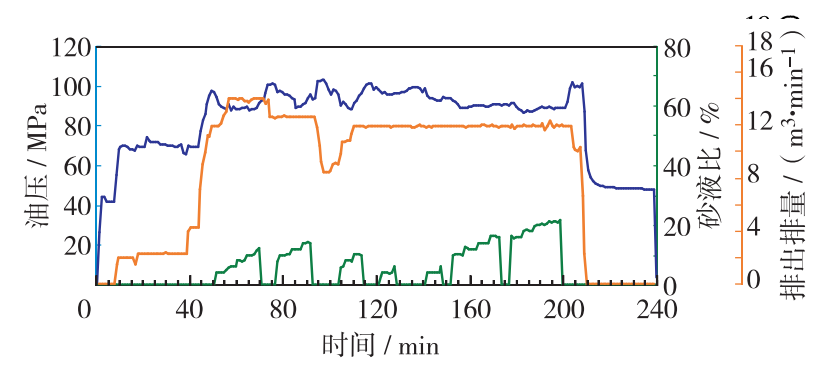

图 11 单级迂回暂堵压裂施工曲线实例

Fig. 11 A case of fracturing pressure curve with temporary plugging treatment

\section{2 多级迂回暂堵实例分析}

涪陵某口示例井段的两级迁回暂堵压裂施工曲 线见图 12. 由图 12 可见, 该段厡注前置液后将排 量提至 $12.6 \mathrm{~m}^{3} / \mathrm{min}$, 洜人第 1 个粉砂段塞时压力 由 $91 \mathrm{MPa}$ 升至 $102 \mathrm{MPa}$, 采取第 1 级迁回降排量至 $11.2 \mathrm{~m}^{3} / \mathrm{min}$ 控制后, 压力降至 $75 \mathrm{MPa}$; 之后在泵 人第 2 个粉砂段塞过程中提排量至 $12 \mathrm{~m}^{3} / \mathrm{min}$, 排 量恢复程度 $95.2 \%$. 砂塞加完后压力又升至 103 $\mathrm{MPa}$ (推测小粒径支撑剂在裂缝端部至中部行成暂 堵), 缝内净压力增加了 $25 \sim 28 \mathrm{MPa}$, 增速约为 $5.2 \mathrm{MPa} / \mathrm{min}$, 采取第 2 级迁回控制降排量至 10.7 $\mathrm{m}^{3} / \mathrm{min}$ 后, 压力从 $77 \mathrm{MPa}$ 恢复到 $86 \mathrm{MPa}$, 缝内净 压力增加了 $8 \sim 9 \mathrm{MPa}$; 之后阶梯升至最高排量 $14.2 \mathrm{~m}^{3} / \mathrm{min}$, 后续施工顺利. 第 2 次排量恢复程

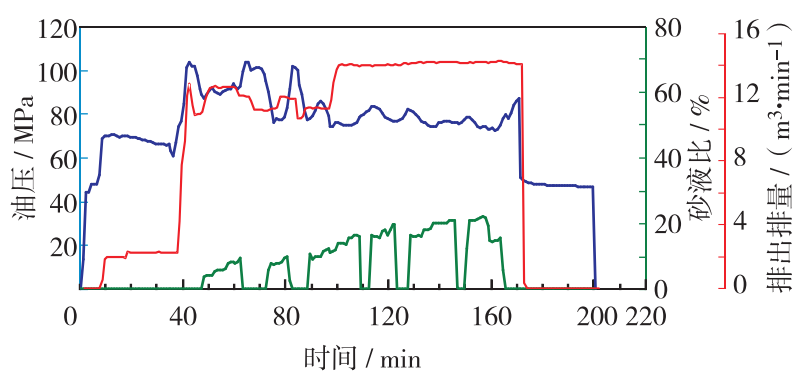

图 12 多级迁回暂堵压裂施工曲线实例

Fig. 12 A case of fracturing pressure curve with circuitous temporary plugging treatment 
度 $112.7 \%$ 。通过 2 次迂回提排量, 较单级迂回提 排量, 净压力增幅明显.

\section{5 结 论}

1）针对深层压裂采用暂堵施工时压力窗口受 限的局限性，以最大限度提高裂缝复杂性及整体改 造体积为目标函数，提出多级迂回暂堵压裂技术的 概念及实现途径，并进行相应的压裂工艺参数模拟 优化研究, 得出了规律性认识.

2）现场应用实例表明，与单级迁回暂堵压裂 技术相比，多级迂回暂堵压裂技术可实现更大的净 压力增幅及裂㖓改造体积增幅，再在从近井筒到缝 端的逐级暂堵技术的配合下，可实现裂缝复杂性及 改造体积的最大化.

3）现场多口井应用效果证明，只要排量降低 幅度小于 $4 \mathrm{~m}^{3} / \mathrm{min}$ ，即使经过更多次的迂回降排量 和提排量, 对套管变形的影响不大. 因此, 多级迁 回暂堵压裂技术具有推广应用价值.

4) 建议进一步扩大多级迂回暂堵压裂技术的 应用规模以验证效果. 同时，可对更多级的迂回暂 堵压裂技术进行试验，以实现在防套变前提下的裂 缝复杂性及改造体积的最大化和经济最优化.

基金项目：国家自然科学基金资助项目（U19B6003）；中石化科技 攻关资助项目 (P20046-3)

作者简介：蒋廷学（1969-)，中国石化集团公司首席专家，正高 级工程师. 研究方向: 储层改造技术研究及应用. E-mail : jiangtx.sripe@ sinopec.com

引文: 蒋廷学, 市晓冰. 深层油气藏多级迁回暂堵压裂技术研 究 $[\mathrm{J}]$. 深圳大学学报理工版, $2021,38(6): 590-597$.

\section{参考文献 / References:}

[ 1 ] SHAHRI M P, HUANG Jian, SMITH C S, et al. An engineered approach to design biodegradables solid particulate diverters: jamming and plugging $[\mathrm{C}] / / \mathrm{SPE}$ Annual Technical Conference and Exhibition. San Antonio, USA: Society of Petroleum Engineers, 2017: SPE-187433-MS. doi : 10. 2118/187433-MS

[ 2 ] JESUS B, CHRISTIAN C, MATTHEW C J, et al. Increased cluster efficiency and fracture network complexity using degradable diverter particulates to increase production: permian basin wolfcamp shale case study $[\mathrm{C}] / /$ SPE Annual Technical Conference and Exhibition. San Antonio, USA: Society of Petroleum Engineers, 2017: SPE-187218-MS. doi :10. 2118/187218-MS
[ 3 ] 龚 蔚, 袁灿明. 多级暂堵转向技术在高石梯一一磨 溪储层改造中的应用 $[\mathrm{J}]$. 天然气地球科学, 2017,28 (8) : 1269-1273.

GONG Wei, YUAN Canming. Application of multistage temporary plugging in Gaoshiti-Moxi reservoir $[\mathrm{J}]$. Natural Gas Geoscience, 2017, 28 (8) : 1269-1273. (in Chinese)

[ 4 ] 苏良银, 常 笃, 齐 银, 等. 超低渗油藏老井体积压 裂技术研究与应用 $[\mathrm{J}]$. 钻采工艺, 2020,42 (2)：7577.

SU Liangyin, CHANG Du, QI Yin, et al. Research and application of SRV fracturing technology for old wells in untra-low permeability reservoir $[\mathrm{J}]$. Drilling \& Production Technology, 2020, 42(2): 75-77. (in Chinese)

[ 5 ] 杨兆中, 易良平, 李小刚, 等. 致密储层水平井段内多 簇压裂多裂缝扩展研究 $[\mathrm{J}]$. 岩石力学与工程学报, 2018, 37(增刊 2) : 73-81.

YANG Zhaozhong, YI Liangping1, LI Xiaogang, et al. Study on multiple-fracture extension within a stage in horizontal well of tight reservoir $[\mathrm{J}]$. Chinese Journal of Rock Mechanics and Engineering, 2018, 37 (Suppl. 2) : 7381. (in Chinese)

[6]曾庆砧, 庄 茁, 柳占立, 等. 页岩水力压裂中多簇裂 缝扩展的全耦合模拟 $[\mathrm{J}]$. 计算力学学报, 2016, 37 (4) : 643-648.

ZENG Qinglei, ZHUANG Zhuo, LIU Zhanli, et al. Fully coupled modeling for multiple clusters growth of hydraulic fractures in shale $[\mathrm{J}]$. Chinese Journal of Computational Mechanics, 2016, 37(4) : 643-648. (in Chinese)

[ 7 ] 时 贤, 程远方, 常 金金, 等. 页岩气水平井段内多簇 裂缝同步扩展模型建立与应用 $[\mathrm{J}]$. 石油钻采工艺， 2018, 40(2) : 247-252.

SHI Xian, CHENG Yuanfang, CHANG Xin, et al. Establishment and application of the model for the synchronous propagation of multi-cluster fractures in the horizontal section of shale-gas horizontal well $[\mathrm{J}]$. Oil Drilling \& Production Technology, 2018, 40 ( 2 ): 247-252. (in Chinese)

[ 8 ] 程远方, 吴百烈, 李 娜, 等. 煤层压裂裂缝延伸及影 响因素分析 $[J]$. 特种油气藏, 2013, 20(2): 126-129. CHENG Yuanfang, WU Bailie, LI Na, et al. Analysis of hydraulic fracture extension and influencing factors in coal seam $[\mathrm{J}]$. Special Oil and Gas Reservoirs, 2013, 20 (2) : 126-129. (in Chinese)

[9]赵金洲, 陈曦宇, 李勇明, 等. 水平井分段多簇压裂模 拟分析及射孔优化 $[\mathrm{J}]$. 石油勘探与开发, 2017, 44 (1) : 117-124. 
ZHAO Jinzhou, CHEN Xiyu, LI Yongming, et al. Numerical simulation of multi-stage fracturing and optimization of perforation in a horizontal well $[\mathrm{J}]$. Petroleum Exploration and Development, 2017, 44 (1): 117-124. (in Chinese)

[10] 郭亚兵. 致密砂岩气藏暂堵转向压裂技术研究 [D]. 成都: 西南石油大学, 2016.

GUO Yabin. Temporary plugging reorientation fracturing in tight sandstone reservoirs [D]. Chengdu: Southwest Petroleum University, 2016. (in Chinese)

[11] YIN Junlu, LIU Huan, CHI Xiaoming, et al. Experimental study and field test of degradable fiber based temporary plugging and diversion fracturing technology $[\mathrm{J}]$. Natural Gas Exploration and Development, 2017, 40 (3) : 113119.

[12] SIDDHAMSHETTY P, WU K, KWON J S. Modeling and control of proppant distribution of multi-stage hydraulic fracturing in horizontal shale wells $[\mathrm{J}]$. Industrial \& Engineering Chemistry Research, 2019, 58 (8) : 31593169.

[13] 段 华, 李荷婷, 代俊清, 等. 深层页岩气水平井 “增 净压、促缝网、保充填” 压裂改造模式一以四川盆 地东南部丁山地区为例 $[\mathrm{J}]$. 天然气工业, 2019, 39 (2) : 72-76.

DUAN Hua, LI Heting, DAI Junqing, et al. Horizontal well fracturing mode of "increasing net pressure, promoting network fracture and keeping conductivity" for the stimulation of deep shale gas reservoirs : a case study of the Dingshan area in SE Sichuan Basin $[\mathrm{J}]$. Natural Gas Industry, 2019, 39(2): 72-76. (in Chinese)

[14] SIDDHAMSHETTY P, WU Kan, KWON J S. Optimization of simultaneously propagating multiple fractures in hydraulic fracturing to achieve uniform growth using databased model reduction $[\mathrm{J}]$. Chemical Engineering
Research \& Design, 2018, 136: 675-686.

[15] PANJAITAN M L, MORIYAMA A, MCMILLAN D, et al. Qualifying diversion in multi clusters horizontal well hydraulic fracturing in haynesville shale using water hammer analysis, step-down test and microseismic data [C]// SPE Hydraulic Fracturing Technology Conference and Exhibition. The Woodlands, USA: Society of Petroleum Engineers, 2018: SPE-189850-MS. doi :10. 2118/189850-MS

[16] 江 旳, 许国庆, 石 阳, 等. 致密岩心带压渗吸的 影响因素实验研究 $[\mathrm{J}]$. 深圳大学学报理工版, 2020, 37(5) : 497-506.

JIANG Yun, XU Guoqing, SHI Yang, et al. Experimental study on influencing factors for forced imbibition in tight sandstone cores $[\mathrm{J}]$. Journal of Shenzhen University Science and Engineering, 2020, 37 ( 5 ) : 497-506. (in Chinese)

[17] 王会敏, 金 珊. 川东南五峰-龙马溪组页岩气勘探开 发进展、主要问题及对策 $[\mathrm{J}]$. 中国矿业, 2019, 28 (9) : 136-142.

WANG Huimin, JIN Shan. Progress, main problems and countermeasures of the shale gas exploration and development in Wufeng-Longmaxi formation, southeast Sichuan [J]. China Mining Magazine, 2019, 28 (9) : 136-142. (in Chinese)

[18] 曾凌翔. 一种页岩气水平井均匀压裂改造工艺技术的 应用与分析 $[\mathrm{J}]$. 天然气勘探与开发, 2018, 41 (3): 99-105.

ZENG Lingxiang. Uniform fracturing technologies and their application to shale-gas horizontal wells $[\mathbf{J}]$. Natural Gas Exploration and Development, 2018, 41(3): 99-105. (in Chinese)

【中文责编：晨 兮; 英文责编：天 澜】 